\title{
SCATSAT-1 Scatterometer data processing
}

\author{
Devang Mankad ${ }^{1, *}$, Rajesh Sikhakolli ${ }^{2}$, Puja Kakkar ${ }^{1}$, Qamer Saquib ${ }^{1}$, \\ Krishna Murari Agrawal ${ }^{1}$, Suresh Gurjar ${ }^{1}$, Dinesh Kumar Jain ${ }^{1}$, \\ V. M. Ramanujam ${ }^{1}$ and Pradeep Thapliyal ${ }^{1}$ \\ ${ }^{1}$ Space Applications Centre, ISRO, Ahmedabad 380 015, India \\ ${ }^{2}$ National Remote Sensing Centre, ISRO, Hyderabad 500 625, India
}

SCATSAT-1 carries a Ku-band scatterometer with a scanning pencil beam configuration. It deploys two beams, a vertically polarized outer beam and a horizontally polarized inner beam, to cover a swath of $1800 \mathrm{~km}$. The mission mainly caters to oceanographic applications and weather forecasting, with the data being extensively used for cyclogenesis predictions across the globe and specifically, the tropical region. Since the launch of SCATSAT-1 in September 2016, the satellite and payload performances as well as mission and ground segment operations have been found to be nominal and satisfactory. This article highlights various levels of operational data products as well as algorithms used for deriving radar backscatter and retrieving wind vector data from scatterometer measurements.

Keywords: Data products, footprint, scatterometer, slices, wind vector.

\section{Introduction}

SCATTEROMETERS are microwave radar instruments designed specifically to enable estimation of near-surface wind velocity (both speed and direction) over the ocean under all-weather conditions. The estimation of ocean surface wind vectors is done from measurements of backscatter cross-section (also known as sigma-naught or sigma0, symbol: $\sigma_{0}$ ) of the sea surface at moderate incidence angles, where the ocean backscatter is dominated by Bragg scattering. India's first scatterometer, viz. OSCAT was launched on-board Oceansat-2 on 23 September 2009. Post launch, the instrument acquired valuable data of ocean wind vectors and was assimilated by various operational weather forecasting agencies like EUMETSAT, NOAA, KNMI, etc.

In the course of regular operations, the instrument provided invaluable data that helped in tropical/global cyclogenesis predictions like Laila (2010) in the Bay of Bengal, Irene (2011) and Sandy (2012) in the Atlantic Ocean and Phailin (2013). OSCAT served the international community until February 2014 (refs 1-4).

\footnotetext{
*For correspondence. (e-mail: devang@sac.isro.gov.in)
}

Launched on 26 September 2016, the SCATSAT-1 mission is continuing the legacy of OSCAT, for the operational generation of wind vector products as well as cyclogenesis predictions. The data quality is also significantly improved for usage in climate records.

The experience of OSCAT processing brought home various lessons for all the teams. While OSCAT payload had a simplified on-board signal processor, the one in the SCATSAT-1 payload is equipped with features such as programmable pick-up of samples - at both signal-plusnoise $(\mathrm{S}+\mathrm{N})$ and noise-only levels, overlapped binning logic and higher precision output (32 in SCATSAT-1 against 16 in OSCAT) to prevent saturation over snow. Cross-patching of main and redundant chains of relevant subsystems has been the vital feature of the improved payload design. The output of on-board signal processor is stored on a solid state recorder and the recorded data are dumped on the ground at different receiving stations situated mainly at the poles. These data are then transferred to the Integrated Multi-mission Ground segment for Earth Observation Satellites (IMGEOS) environment at National Remote Sensing Centre (NRSC), Shadnagar, Telangana. Further, the data are decoded on ground to generate time-tagged, scan-wise formatted sensor data along-with corresponding orbit and attitude information. Using these inputs, the SCATSAT-1 data processing software generates various levels of products that are disseminated to the national and international community of users in required timelines. The levels of products have been identified at par with similar international previous and current missions. Here we describe various levels of products and algorithmic approaches followed in order to generate these products.

\section{Levels of SCATSAT-1 data products}

The SCATSAT-1 return signal is demodulated, decimated and sampled to form $4 \mathrm{~K}$ complex raw echo samples. The on-board processor carries out signal processing operations to compress the data in range direction and generates $\mathrm{S}+\mathrm{N}$ and noise-only samples depending on the mode of operation.

Thus, sensor echo data that serve as one of the inputs for further data product generation contains the following: 
(a) Slice data comprising $\mathrm{S}+\mathrm{N}$ samples, wherein each slice consists of the sum of powers of a pre-configured number of samples of the deramped echo taken in the frequency domain. (b) Noise data, which typically are the sum of powers of the lower and upper noise sidebands. The noise samples are used to estimate the actual signal power from the $\mathrm{S}+\mathrm{N}$ data. (c) Loopback calibration data that are a measure of transmit power used. (d) Auxiliary data inserted by data acquisition and compression system (DACS) and payload controller (PLC) subsystems of the payload.

Apart from the echo data, orbit and attitude data consisting of the satellite state vectors, attitude information and other ancillary parameters form vital inputs. As the rotation speed of the payload scan mechanism is fixed nominally at $20.5 \mathrm{rpm}$, every scan of the echo data is available at an interval of approximately $2.93 \mathrm{sec}$. Post processing on the ground, orbit and attitude data is available every second.

Various levels of data products are defined for SCATSAT-1. These include:

(1) Level-1B (L1B) data product: This is a representative of the actual scan data. The number of $\mathrm{S}+\mathrm{N}$ samples is 40 for the nominal mode of operation. A subset of the $\mathrm{S}+\mathrm{N}$ sample set, typically 15 for the outer beam and 9 for the inner beam, represents return signal from within $3 \mathrm{~dB}$ antenna footprint and qualifies as a slice. Input count data received for $\mathrm{S}+\mathrm{N}$ and noise-only channels are converted to power for these candidate slices. Data from the slices are aggregated to form a footprint. Relevant parameters for each slice and footprint within a scan are computed and packed to form a L1B product. Appropriate flagging of slice and footprint data is done, based on observations on land, sea, coastal boundaries and ice regions. For each ascending and descending pass, the product is generated and disseminated to the user community. L1B products are utilized extensively by advanced users to generate nominal and high-resolution wind products.

(2) Level-2A (L2A) data product: As L1B product represents the way in which the scatterometer has scanned the earth, there are overlaps across footprints within multiple scans. To simplify interpretation of the dataset, mapping of a set of observations to a geographical area becomes essential. For each half-orbit, i.e. data imaged from North Pole to South Pole or vice versa, subsatellite points at a fixed grid interval $(25$ or $50 \mathrm{~km})$ are obtained using satellite ephemeris. Using outer beam swath information, extreme points of swath corresponding to these sub-satellite points are obtained. The intermediate points of swath grid are obtained using viewing and earth geometry to derive the wind vector cells (WVCs). Various fore and aft observations are co-located in the grid cells and $\sigma_{0}$ is provided for each of these cells. L2A data products are used to generate wind data that are further utilized in various assimilation models by the weather forecasting community.
(3) Level-2B (L2B) data product: This is generated by using $\sigma_{0}$ in swath grid as inputs, along with other wind model-related information. Co-located $\sigma_{0}$ observations of L2A product along with geophysical model function (GMF) are used to generate wind vector information corresponding to each cell of the swath grid. Wind vectors are marked for sea-ice and rain flags.

(4) Level-3 data products: While level-2 products are defined in the form of a swath grid and are available for every half-orbit (ascending and descending passes), day wise sigma-naught and wind products are generated by combining L2A and L2B products respectively. Both products are generated at a grid spacing of $0.25^{\circ}$ and $0.5^{\circ}$ in both directions. Level-3 $\sigma_{0}$ (L3S) product is obtained by averaging all L2A observations falling within a grid cell. In case of level-3 wind (L3W) product, all observations are mapped to a particular grid cell and the latest observation is assigned to the grid cell.

\section{Processing algorithms for derivation of $\sigma_{0}$}

Derivation of scan-mode $\sigma_{0}$ consists of the following two components: (1) Computation of $\sigma_{0}$ using radar equation. (2) Computation of geo-location of slices.

\section{Computation of $\sigma_{0}$}

The total power received $\left(P_{\mathrm{r}}\right)$ by the sensor consists of backscattered power $\left(P_{\mathrm{b}}\right)$ by target and additional noise introduced by the instrument electronics $\left(P_{\mathrm{n}}\right)$. The relation between normalized radar backscatter, $\sigma_{0}$ of the target and power transmitted $\left(P_{\mathrm{t}}\right)$ and backscattered by the radar ${ }^{5}$ is written as

$$
P_{\mathrm{b}}=\frac{\lambda^{2}}{(4 \pi)^{3}} \int_{\text {area }} \frac{P_{\mathrm{t}} G^{2} \sigma_{0}}{R^{4}} \mathrm{~d} A,
$$

where $\lambda$ is the wavelength, $G$ the antenna gain, $R$ the range and $\mathrm{d} A$ denotes the illuminated area. We can relate $\sigma_{0}$ to $P_{\mathrm{b}}$ using $x$ factor (or $X$ ) as

$$
\sigma_{0}=\frac{P_{\mathrm{b}}}{X},
$$

where $X$ is defined as

$$
X=\frac{\lambda^{2}}{(4 \pi)^{3}} \int_{\text {area }} \frac{P_{\mathrm{t}} G^{2}}{R^{4}} \mathrm{~d} A .
$$

Antenna gain parameter can be deduced from antenna pattern data. To speed up computations, the antenna gain components are summed over azimuth angles and stored as look-up tables. Due to the instrument thermal noise 
and radar signal fading effects, the estimates of $\sigma_{0}$ will be noisy. A parameter commonly used to indicate the magnitude of the noise is $K p$, which is defined as the normalized standard deviation of the echo return energy ${ }^{6}$. It is a function of instrument signal processing parameters and the return signal-only to noise-only energy ratio (SNR). Power computed for $\mathrm{S}+\mathrm{N}$ samples and noise-only samples are used to deduce signal-only power, and SNR is further computed using the same. The equation defining $K p$ is given below.

$$
\begin{aligned}
& K p_{\mathrm{a}_{\text {slice }}}=\frac{1}{\text { freq }_{\mathrm{bin}_{\mathrm{m}}} * t_{\mathrm{p}}}, \\
& K p_{\mathrm{b}_{\text {slice }}}=\frac{2}{\text { freq }_{\mathrm{bin}_{\mathrm{m}}} * t_{\mathrm{g}}}, \\
& K p_{\mathrm{c}_{\text {slice }}}=\frac{1}{\text { freq }_{\mathrm{bin}_{\mathrm{m}}} * t_{\mathrm{g}}} *\left(1+\frac{\text { freq }_{\mathrm{bin}_{\mathrm{m}}}}{\text { freq }_{\text {noise }_{\mathrm{se}}}}\right), \\
& K p_{\text {slice }}=\left(K p_{\mathrm{a}_{\text {slice }}}+\frac{K p_{\mathrm{b}_{\text {slice }}}}{\mathrm{SNR}_{\text {slice }}}+\frac{K p_{\mathrm{c}_{\text {slice }}}}{\mathrm{SNR}_{\text {slice }}^{2}}\right)^{1 / 2},
\end{aligned}
$$

where $t_{\mathrm{p}}$ is the pulse width, $t_{\mathrm{g}}$ the receive window time, freq $_{b_{\text {in }}}$ the size of frequency bin for mode $m$, freq $q_{\text {noise }}$ the frequency bandwidth for noise and $\mathrm{SNR}_{\text {slice }}$ is the SNR for the slice.

Brightness temperature for slices and footprints is derived using noise-only power.

\section{Geo-locating slices and footprints}

The SCATSAT- 1 footprints are of size $27 \mathrm{~km} \times 46 \mathrm{~km}$ for the inner beam and $30 \mathrm{~km} \times 70 \mathrm{~km}$ for the outer beam. It acquires data of $H H$ polarization for the inner beam and $V V$ polarization for the outer beam at two look angles. Based on the knowledge of position and velocity vectors of the platform, look angles, scan angle, attitude components and other ancillary information, the geolocation of footprint centres is computed. Based on the knowledge of antenna pattern beamwidth, the geolocation of the slice centres is computed.

\section{Generation of swath grid and cell posting}

To generate swath grid for co-located backscatter observations, satellite position and velocity parameters are used. Using the latitude and longitude of each footprint centre for all fore and aft observations, the footprints are assigned to the respective grid cells along with relevant geometric parameters. The swath is defined with respect to satellite ground trace and divided into cells of approx- imately equal area $(25 \mathrm{~km} \times 25 \mathrm{~km})$ in both across- and along-track directions. A grid of non-overlapping cells is thus generated for every half-orbit of data. Subsequently, the cells are populated with all footprints from scan mode data for which the corresponding footprint centres lie within the defined cell. At a finer level, for each footprint, the slice centres are analysed for their location within the given cell boundaries. Contributions of multiple slices from the same footprint are aggregated and appropriately averaged to form a composite. All the composites in a WVC are used to derive the wind vector. For this, information about look (fore/aft), $\sigma_{0}$ quality and surface features (land/sea/ice) is essential. This is embedded in individual bits of a 16-bit word associated with all composites.

\section{Wind retrieval process}

The normalized radar backscatter also known as $\sigma_{0}$, is the basic parameter measured by a scatterometer, which is directly proportional to the ocean surface roughness generated by the action of wind field through Bragg resonance principle ${ }^{5}$. By measuring $\sigma_{0}$ from different azimuth directions, it is possible to estimate both wind speed and direction of a given region on the ocean surface ${ }^{7-10}$. Retrieval of ocean surface wind vector from spaceborne pencil-beam microwave scatterometer generally consists of the following important components: (1) Development of GMF, which is the most crucial element for wind vector retrieval. (2) Development of retrieval algorithms that involve point-wise extraction of prioritized wind vector solutions from radar backscatter data by making use of suitable GMF. (3) Development of techniques for wind directional ambiguity removal over the entire swath divided into three regions, namely nadir, middle/sweet swath and outer swath regions. The ambiguity removal specifically in the outer swath region requires auxiliary data of wind fields from models in the form of forecast/analysis. (4) Development of quality control procedures for flagging out data under rainy and other ambiguous conditions. In this study the algorithms developed and implemented for SCATSAT GMF development, wind retrieval, ambiguity removal and rain flagging will be presented along with a limited validation of retrieved vector winds using European Center for Medium range Weather Forecasting (ECMWF) analysed winds.

\section{GMF development}

GMF is the relationship between ocean surface wind vector (speed and direction) and the oceanic radar backscatter observed at a particular radar frequency, polarization and observational geometry. This mathematical relationship also known as empirical model function, is established empirically using global satellite radar backscatter 
observations and the corresponding ocean surface wind vector obtained from various sources like reanalysis from global atmospheric numerical weather prediction (NWP) models, in situ data from ships and buoys and other satellites for a desired period of time ${ }^{11}$. Ideally the GMF coefficients should have been developed using accurate wind information typically coming from buoy measurements. Due to the limited coverage of buoy measurements, practically it takes many years to get the entire dynamic range of winds covered. Hence, the GMF is generally developed post-facto using collocated wind observations mostly from the NWP model and scatterometer observations over a period of several months covering the full dynamic range of the wind vector. The main advantage of using NWP-based analysis winds for developing GMF is that within a limited period of few months, we can get the collocated observations covering the entire dynamic range of wind speeds and directions to model $\sigma_{0}$ variability. Exploiting this major advantage since QuikSCAT period, it became a practice to use NWP model winds for development of GMF coefficients. However, one basic disadvantage is that if the NWP model winds used have any systematic errors, they can affect GMF coefficients and in turn the retrieved winds. To overcome this, fine-tuning of the GMF coefficients based on the validation results of the retrieved winds with various in situ and contemporary satellite winds during the preoperational period is also carried out before finalizing the GMF coefficients specific to a particular scatterometer system. The functional form of GMF relating the scatterometer-measured $\sigma_{0}$ and wind vector is generally written as

$$
\sigma_{0}=A_{0}\left[1+A_{1} \cdot \cos (\varphi)+A_{2} \cdot \cos (2 \varphi)+A_{3} \cdot \cos (3 \varphi)\right],
$$

where the coefficients $A_{i}$ are functions of wind speed, incidence angle and polarization. In order to determine the coefficient $A_{0}$ at a desired incidence angle, wind speed and polarization, the $\sigma_{0}$ values are averaged (in $\mathrm{dB}$ scale) over relative wind direction $\varphi$ varying from 0 to $2 \pi$ yielding $A_{0}$ coefficient. Following the methodology used for OSCAT GMF development described in ref. 12, SCATSAT-specific GMF is developed, for which we have used eight months of SCATSAT L2A $\sigma_{0}$ observations from 15 October 2016 to 15 July 2017, processed with the latest version (Version 1.1.3) of the algorithms. The L2A data which have been flagged for the presence of ice, poor quality, negative $\sigma_{0}$ or coastal ocean $(50 \mathrm{~km}$ from the coast) are discarded to avoid any contamination. The reference ocean surface vector winds are taken from ECMWF analysed winds ${ }^{13}$ available at $12.5 \mathrm{~km}$ grid and four times a day $(00,06,12$ and 18 GMT). Using spatiotemporal interpolation technique for each L2A $\sigma_{0}$ observation, we have obtained a collocated ECMWF wind speed and direction. This collocated dataset (which has around 80 million points) is divided into different wind speeds $(0-25 \mathrm{~m} / \mathrm{s}$ at an interval of $1 \mathrm{~m} / \mathrm{s})$ and directional bins $\left(0^{\circ}-360^{\circ}\right.$ at an interval of $\left.1^{\circ}\right)$, and for each bin the statistics (mean and standard deviation) of $\sigma_{0}$ variability was calculated. For a given wind speed bin, assuming uniform distribution of collocated data across all the wind direction bins, the $A_{0}$ coefficient is calculated by averaging across all the direction ranges. Subsequently, employing a data-driven enhancement technique for very low $(<4 \mathrm{~m} / \mathrm{s})$ and high winds $(>24 \mathrm{~m} / \mathrm{s})$ described in Gohil et al. ${ }^{12}$ the GMF coefficients $A_{1}, A_{2}$ and $A_{3}$ are calculated yielding a smooth mathematical model termed as GMF.

In Figure 1, we show mean $\sigma_{0}$ for different wind speeds and relative directions from the developed SCATSAT-specific GMF.

\section{Retrieval of prioritized wind vector solutions}

With the dependencies of scatterometer-measured backscatter on wind speed and wind direction being nonlinear (power law) and bi-harmonic in nature respectively, yield multiple solutions of wind vector from a set of backscatter measurements while performing retrieval using $\mathrm{GMF}^{14,15}$. A retrieval algorithm developed in-house earlier, has been applied to fan-beam scatterometers of Seasat ${ }^{16}$ and ERS-1 satellites ${ }^{17}$ using SASS-I (Ku-band) and CMOD-4 (C-band) model functions respectively. An updated version of this algorithm known as the 'normalized standard deviation (NSD)' algorithm ${ }^{18}$ has been developed in-house for retrieving prioritized wind vector solutions from the scatterometer data and used operationally for OSCAT, and the same with due fine-tuning is being used for SCATSAT-1 as well. The algorithm makes use of closure form of relationship between wind speed and backscatter for an aspect direction to retrieve wind speed from a given single backscatter measurement. From a given set of backscatter data for a given wind vector cell, the mean and normalized standard deviation of wind speed are obtained for all the aspect directions. The prioritized wind vector solutions are obtained through searching minima of wind speed NSD along the aspect wind direction and ranked according to the NSD values. The prioritization of solutions is based on the fact that for an aspect wind direction to be the true wind direction, the derived wind speeds from a set of backscatter measurements should be the same, or on the other hand, the NSD value should be zero or minimum. Among the multiple wind direction solutions only one direction is the true direction, while the remaining are ambiguities.

\section{Directional ambiguity removal}

The rank-1 direction is always the true direction for noise-free data cases, while for the real (noisy) data cases the true direction is represented by the first two highest ranking solutions, which may either be true or in almost 
(a)

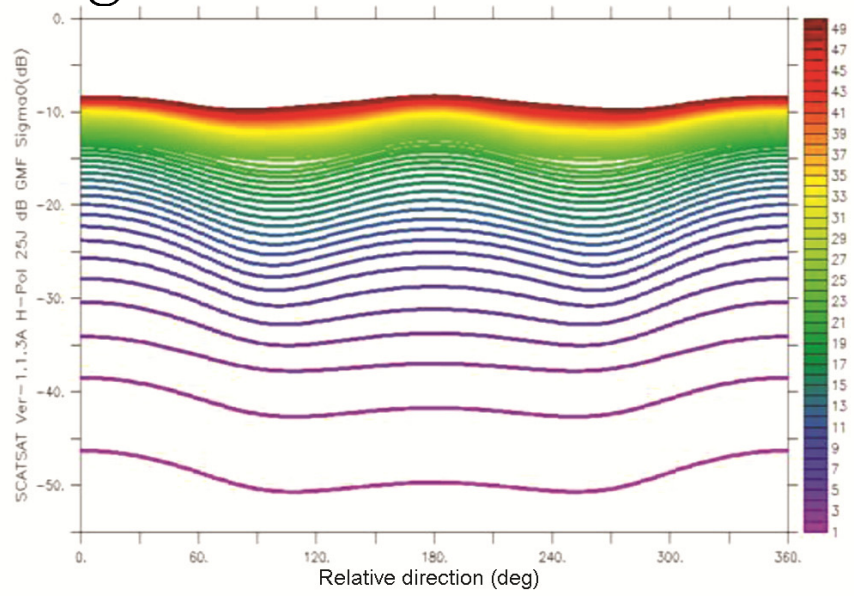

(b)

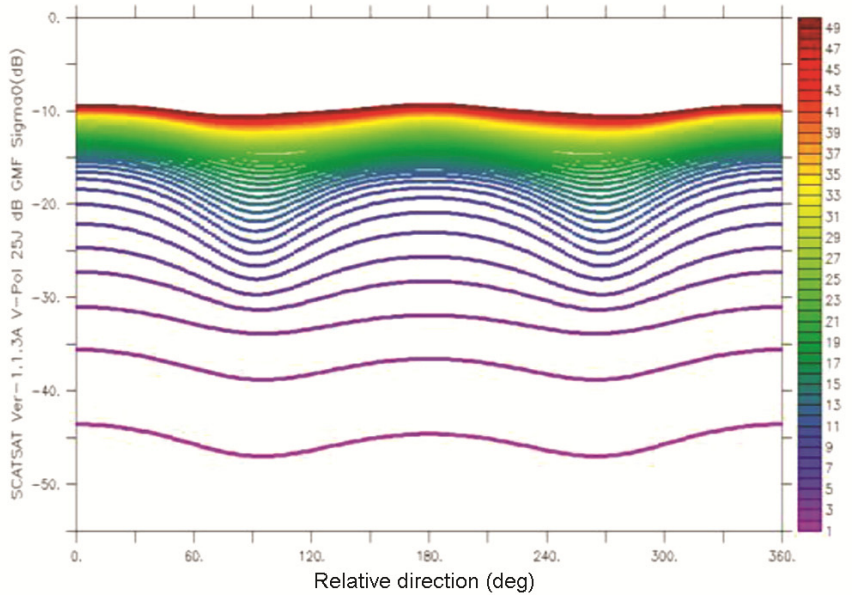

Figure 1. SCATSAT GMF $\sigma_{0}$ for different wind speeds (shown in different colours) and relative directions. $\boldsymbol{a}, H H$ polarization, $\boldsymbol{b}, V V$ polarization.

opposite direction (an ambiguity). This directional ambiguity needs to be filtered out, which is realized through a median filter. Based on the circular median filter and aspects related to directional stability and conservation of scattering (DiSCS), an algorithm has been developed ${ }^{19}$ for extracting directional ambiguity-filtered wind vector field using the wind field consisting of prioritized wind vector solutions. This is the DiSCS algorithm and has been used operationally for OSCAT as well as for SCATSAT. This algorithm procedure begins with a 'nudging' process in which the wind vector solution with direction closest to the collocated NWP model (for SCATSAT we use ECMWF model forecast) direction is taken as the initial guess. Subsequently, using the circular median approach the spatial consistency is verified and finally a field of wind vectors which are consistent with each other is obtained to form the final wind vector for each WVC. The physical basis for this approach is that the natural directional variability is very high for low winds $(<3 \mathrm{~m} / \mathrm{s})$ and also the retrieval skill for the low winds and very high winds is relatively small. Exploiting these unique characteristics, a new directional stability weighting is introduced for improving the directional accuracy for the retrieved winds. The directional ambiguity filtering is first applied to only rain-free cases and then to the rain-affected wind vectors utilizing directional information from the neighbouring rain-free cases in order to improve the wind directional flow. Once the wind direction is modified and finalized during the ambiguity removal process, the wind speed is again updated using GMF and the observed backscatter, conserving the relationship between the retrieved wind vector and the observed backscatter. Again, it is important to note that the retrieval of ambiguity-filtered wind vector field is mainly dependent on the amount of noise present in radar backscatter data as well as on its spatial distribu$\operatorname{tion}^{20}$.

\section{Atmospheric corrections}

The Ku-band radar backscatter is affected by heavy clouds and precipitation in terms of attenuating the transmitted and received powers of the scatterometer. Thus, if such information is available in a suitable manner, the radar backscatter can be corrected for atmospheric attenuation improving the wind vector retrieval. Simulations have been earlier performed to study the impact of atmospheric corrections to ADEOS-1 NSCAT $\mathrm{Ku}$-band scatterometer simulated data for wind vector retrieval under cloudy conditions. However, retrieval of wind vector under rainy conditions becomes difficult due to several reasons. One is the two-way attenuation and the others are backscattering caused by rainfall and changes in ocean surface roughness due to the impact of falling rain drops modifying the oceanic radar backscatter. Due to these reasons, retrieval of wind vector under rainy conditions is normally not performed, but such data are flagged obtaining rain information from other sources like microwave radiometers/radars, or using models of rain attenuation and scattering contributions (developed separately) in processing scatterometer data for any possible rain flagging ${ }^{21,22}$. One such algorithm had been developed during the OSCAT period for identification of rain-affected wind vectors based on dynamical detection of extreme events of brightness temperature (noise only) data and the post-retrieval cost-function ${ }^{12}$. This algorithm with updated threshold conditions tuned for SCATSAT-1 data is used for rain-flagging. Moreover, a new algorithm (which will be communicated in a separate paper) using 


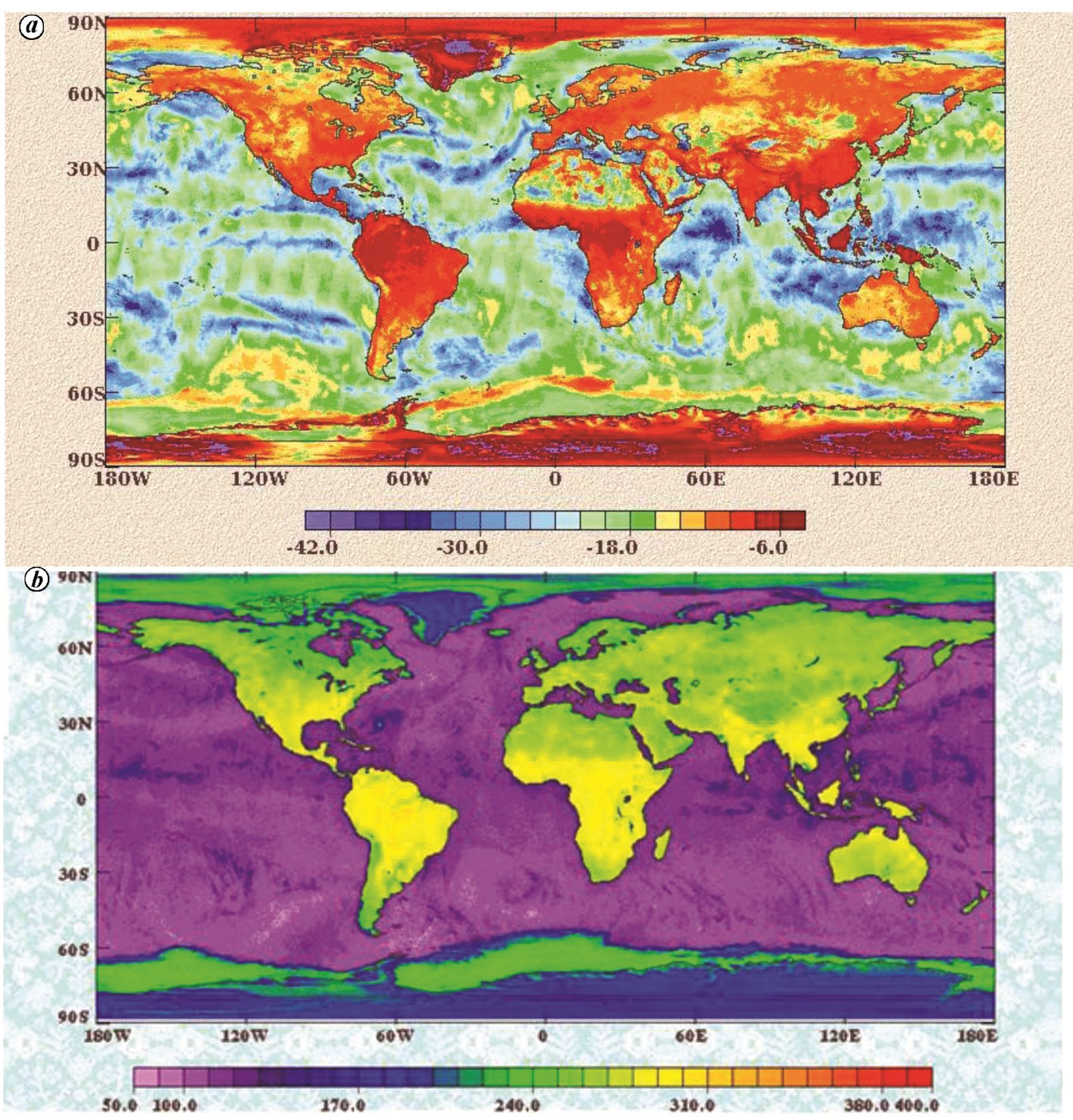

Figure 2. $\boldsymbol{a}, \sigma_{0}(\mathrm{~dB})$ for the outer beam. $\boldsymbol{b}$, Brightness temperature $(\mathrm{K})$ for the inner beam.

Bayesian estimation method has been developed for correcting the rain-affected wind speeds.

\section{Quality control and validation of products}

All the above-mentioned algorithms along with the SCATSAT-1 specific GMF have been made operational at NRSC, Shadnagar for near real-time processing of SCATSAT-1 data. Figure $2 a$ and $b$ demonstrates the global measurements of $\sigma_{0}$ (outer beam) and brightness temperature (inner beam) respectively, for a cycle of 2 days during the initial phase of operations.

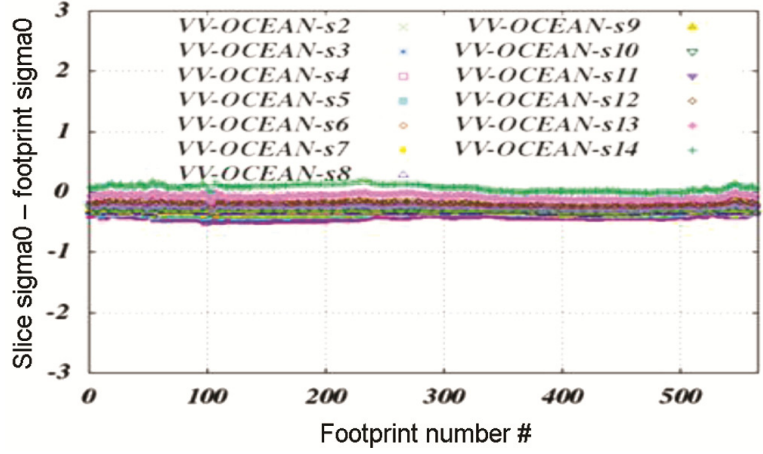

Figure 3. Difference in backscatter with respect to middle slice of a footprint for observations over ocean in the outer beam. 


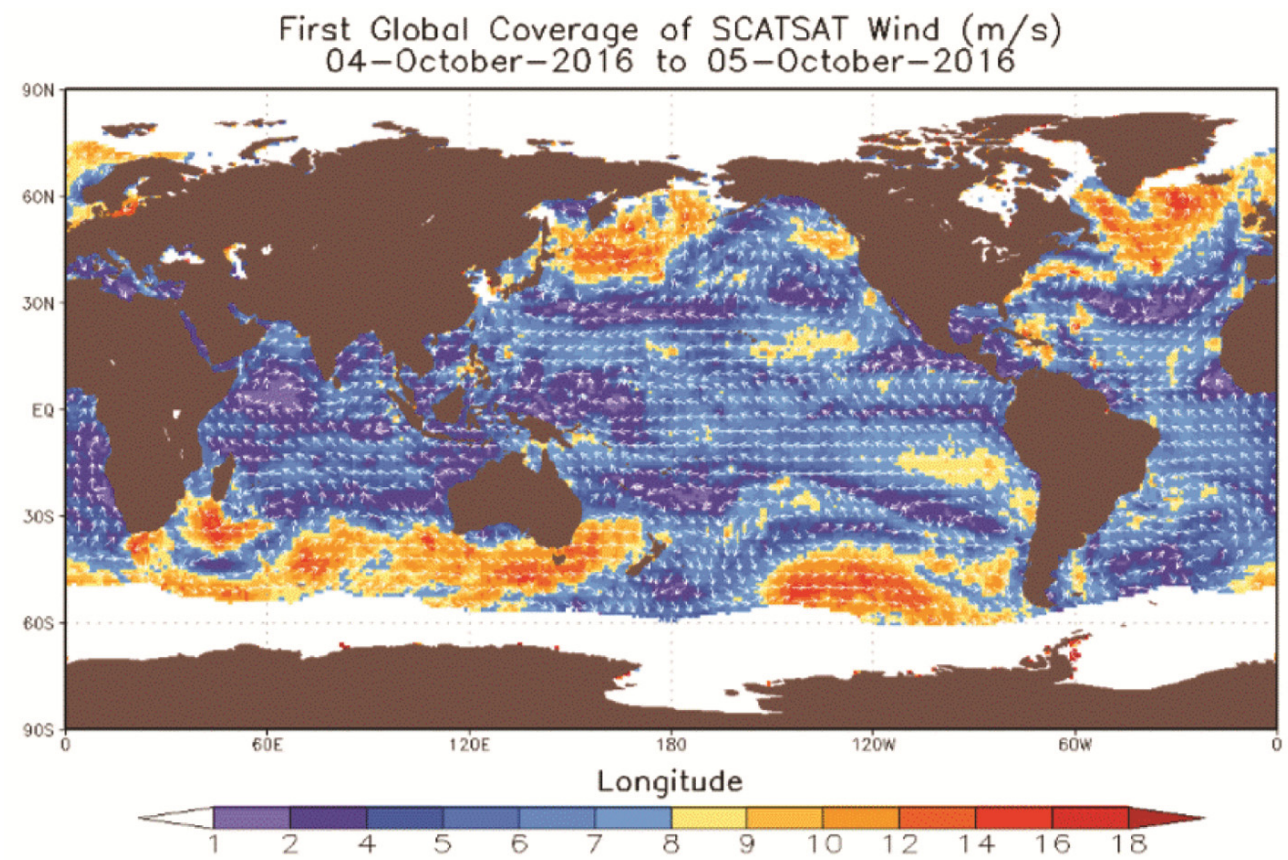

Figure 4. First global coverage of ocean surface wind vectors from SCATSAT-1 observations.

\section{Preliminary validation of backscatter}

Since in on-board compression, not all the Doppler that has acted on the signal has been compensated, $3 \mathrm{~dB}$ of the footprint does not reside at the centre of the input sample array and it is necessary to locate the $3 \mathrm{~dB}$ data peak, which is done using statistical method. Around the data peak, slice data pick-up and their radiometric correction are based on an equi-frequency approach, as the input raw data are periodogram-binned at a specific frequency. Correction for the same can be validated in two ways: (1) slice histograms and (2) residual bias within a footprint.

\section{Slice histograms}

In this method, if the histogram of all slices for any given footprint converges, then it can be stated that the slices are balanced. Histograms over ocean for sample footprints covering the entire scan for the inner and outer beams are studied for multiple months of SCATSAT-1 datasets. It has been observed that the slice balancing is appropriate and as expected.

\section{Residual bias}

It is expected that the difference between slice $\sigma_{0}$ and footprint $\sigma_{0}$ should be the representation of incidence angle variation within a footprint and it should not have any scan angle dependency. From Figure 3, it is clear that for a given footprint, difference in any slice $\sigma_{0}$ and footprint $\sigma_{0}$ is within 0.15 and $0.3 \mathrm{~dB}$ for $H H$ and $V V$ respectively, attributing to their incidence angle variation within the footprint. Additionally, the difference does not have any footprint-dependent variation.

Both results do not show any systematic trend with respect to scanning angle. It shows that all system-induced effects due to its acquisition geometry have been properly taken care during data processing.

\section{Results over standard calibration sites}

Data products quality can be judged by their performance over reference calibration sites like Amazon rainforest, Greenland and Antarctica. Difference in $\sigma_{0}$, for a collocated area, in fore and aft looks of the same pass, should be negligible owing to similar imaging conditions. Also, the difference in ascending and descending passes for a collocated area must be minimum and should be representative of diurnal variation only. From analysis of data products generated so far, it is observed that the differences in backscatter between fore and aft looks for $V V$ and $H H$ beams over the Amazon region are only of the order of $0.05 \mathrm{~dB}$. Differences in other sites are also well within limits.

\section{Preliminary validation of wind data}

In Figure 4, we show the first global coverage of SCATSAT-1 retrieved winds on 4 and 5 of October 2016. During the initial calibration and validation phase, a thorough evaluation of the performance of all these 

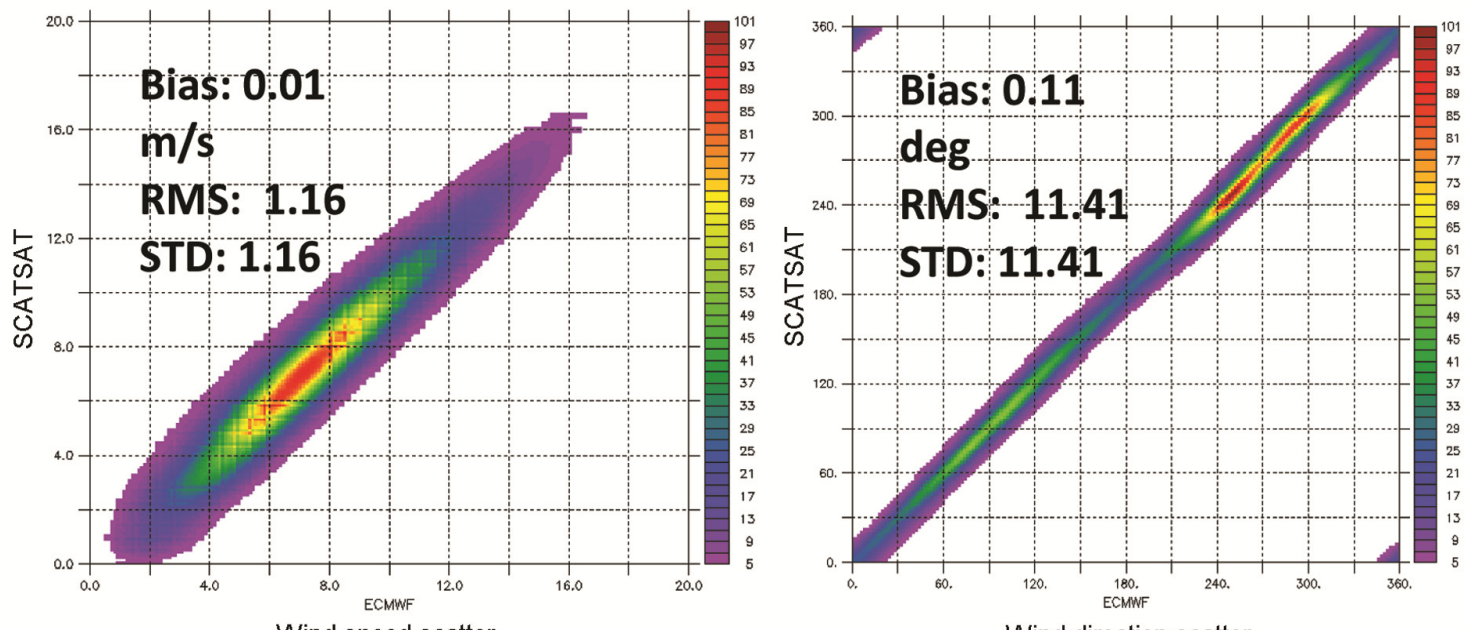

Figure 5. Density scatter plot between SCATSAT-1 retrieved winds and collocated ECMWF analysis winds for the period 15 October 2016 to 15 July 2017.
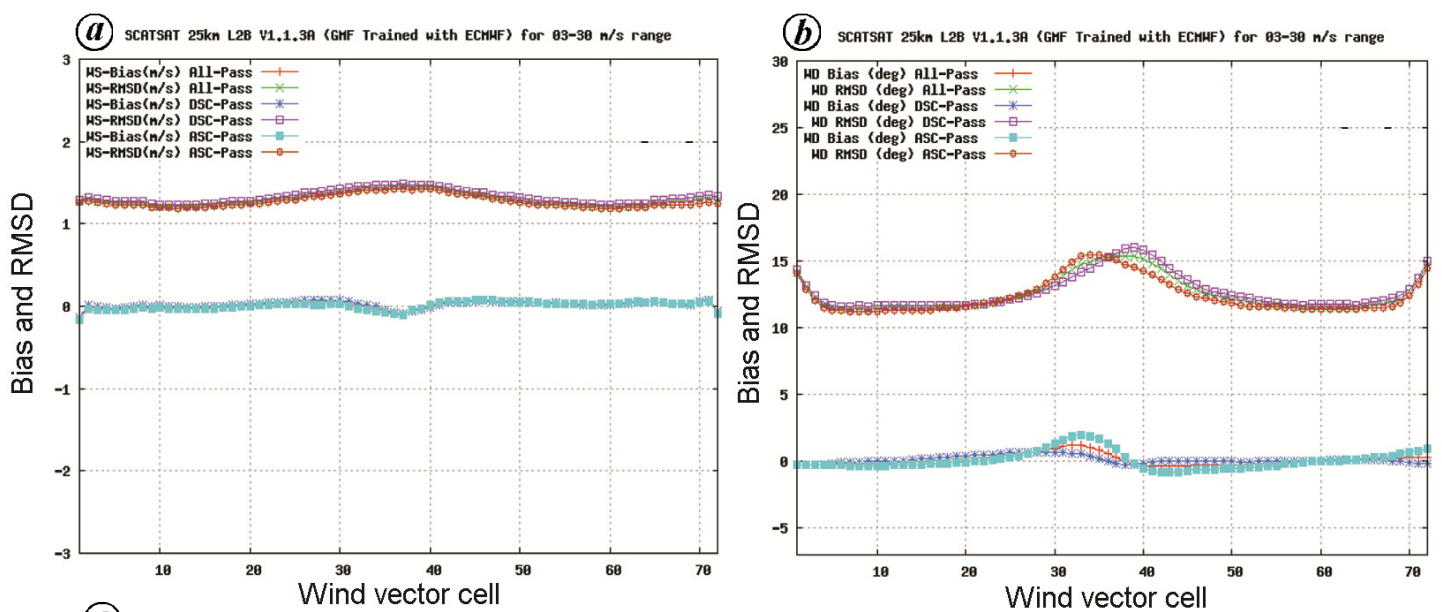

(C) SCATSAт 25kn L28 V1.1.39 Given in Aug, 2017 (GHF Trained with ECHHF)
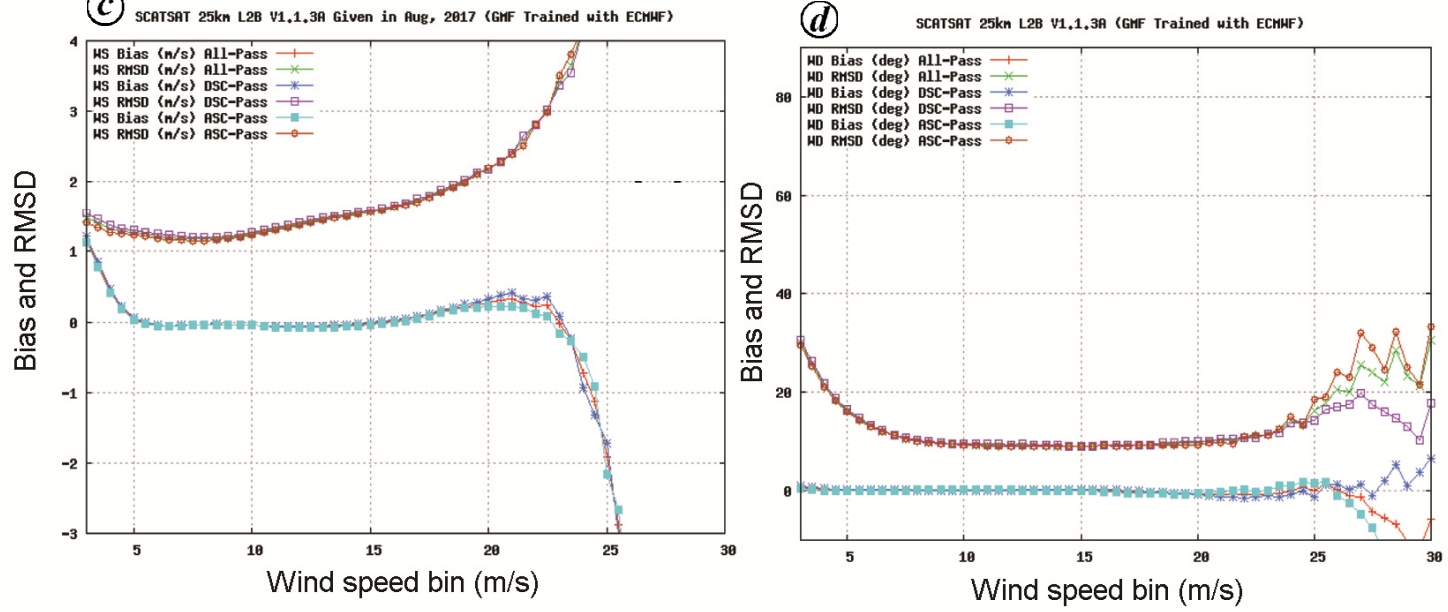

Figure 6. Variation of bias $(\mathrm{m} / \mathrm{s})$ and RMSE $(\mathrm{m} / \mathrm{s})$ between SCATSAT retrieved winds and collocated ECMWF analysis winds as a function of wind vector cell (WVC) number (top row) and collocated ECMWF wind speed bin (bottom row). $\boldsymbol{a}$, For wind speed as a function of WVC number. $\boldsymbol{b}$, For wind direction as a function of WVC number. $\boldsymbol{c}$, For wind speed as a function of ECMWF wind speed bin. $\boldsymbol{d}$, For wind direction as a function of ECMWF wind speed bin.

algorithms has been done and the required fine-tuning/ improvements in the processing algorithms performed which led to substantial improvement in the overall quality of the SCATSAT-1 retrieved winds. In order to demonstrate the quality of SCATSAT-1 retrieved winds, a limited validation of SCATSAT-1 V1.1.3 L2B wind data 
using collocated ECMWF analysis winds for the period of October 2016 to July 2017 is performed.

In Figure 5, we show the density scatter plot between these two wind products, which clearly shows an excellent agreement with a negligible bias and having a root mean square error (RMSE) of $1.16 \mathrm{~m} / \mathrm{s}$ and $11.41^{\circ}$ for wind speed and direction respectively. Figure 6 shows the variation of bias and RMSE as a function of WVC number and ECMWF wind speed bin.

From Figure $6 a$ and $b$ it is clear that the across-track variation in bias is close to zero for all the WVCs and RMS also varies well below the mission specifications of $1.8 \mathrm{~m} / \mathrm{s}$ and $20^{\circ}$ for wind speed and direction respectively, thus making SCATSAT-1 data meet the climate quality requirements. Similarly, Figure $6 c$ and $d$ shows variation of bias and root mean square as a function of ECMWF analysis wind speed bins, which clearly shows that except for low $(<5 \mathrm{~m} / \mathrm{s})$ and high winds $(>20 \mathrm{~m} / \mathrm{s}$; for which ECMWF itself has a high variability), the bias and RMS in both wind speed and direction vary well within the mission specifications. A detailed validation of the SCATSAT retrieved winds with in situ buoy, other scatterometer data like ASCAT and NWP models is presented in a separate paper.

\section{Conclusion}

SCATSAT-1 launched in September 2016 is working satisfactorily. The payload, data products and applications have met the mission objectives, and all operations from data reception to product generation and further dissemination are meeting the required timelines. The data products have met the climate quality standards and are able to cater to international requirements of Scatterometer derived wind vectors to feed into routine weather prediction models.

1. Misra, T. et al., Oceansat-II scatterometer: sensor performance evaluation, $\sigma_{0}$ analyses and estimation of biases. IEEE Trans. Geosci. Remote Sensing, 2014, 52, 3310-3315.

2. Bhowmick, S. A., Kumar, R. and Kiran Kumar, A. S., Cross calibration of the OceanSAT-2 scatterometer with QuikSCAT scatterometer using natural terrestrial targets. IEEE Trans. Geosci. Remote Sensing, 2014, 52, 3393-3398.

3. Kumar, R., Chakraborty, A., Parekh, A., Sikhakolli, R., Gohil, B. S. and Kiran Kumar, A. S., Evaluation of Oceansat-2-derived Ocean surface winds using observations from global buoys and other scatterometers. IEEE Trans. Geosci. Remote Sensing, 2013, 51, 2571-2576.

4. Chakraborty, A., Deb, S. K., Shikakolli, R., Gohil, B. S. and Kumar, R., Intercomparison of OSCAT winds with numericalmodel-generated winds. IEEE Geosci. Remote Sensing Lett., 2013, 10, 260-262.

5. Ulaby, F. T., Moore, R. K. and Fung, A. K., Microwave Remote Sensing-Active and Passive, Vols. 1 and 2, Addison-Wesley Publ. Co, Reading, Mass, USA, 1981.

6. Long, D. G. and Spencer, M., Radar backscatter measurement accuracy for a spaceborne pencil-beam wind scatterometer with transmit modulation. IEEE Trans. Geosci. Remote Sensing, 1997, 35, 102-114.

7. Fischer, R. E., Standard deviation of scatterometer measurements from space. IEEE Trans. Geosci. Electron., 1972, 10, 106-113.

8. Schroeder, L., Boggs, D. H., Dome, G., Halberstam, I. M., Jones, W. L., Pierson, W. J. and Wentz, F. J., The relationship between the wind vector and the normalized radar cross section used to derive Seasat-A satellite scatterometer winds. J. Geophys. Res., 1982, 87, 3318-3336.

9. Pierson Jr, W. J., Probabilities and statistics for backscatter estimates obtained by a scatterometer. J. Geophys. Res., 1989, 94, 9743-9759.

10. Long, D. G. and Mendel, J. M., Identifiability in wind estimation from scatterometer measurements. IEEE Trans. Geosci. Remote Sensing, 1991, 29, 268-276.

11. Wentz, F. J. and Smith, D. K., A model function for the oceannormalized radar cross-section at $14 \mathrm{GHz}$ derived from NSCAT observations. J. Geophys. Res., 1999, 104(11), 499-514.

12. Gohil, B. S., Sikhakolli, R. and Gangwar, R. K., Development of geophysical model functions for Oceansat-2 scatterometer. IEEE Geosci. Remote Sensing Lett., 2013, 10, 377-380.

13. Uppala, S. M. et al., The ERA-40 re-analysis. Q. J. R. Meteorol. Soc., 2005, 131, 2961-3012.

14. Stoffelen, A. and Anderson, D., Scatterometer data interpretation: measurement space and inversion. J. Atmos. Ocean Technol., 1997, 14, 1298-1313.

15. Stoffelen, A. and Portabella, M., On Bayesian scatterometer wind inversion. IEEE Trans. Geosci. Remote Sensing, 2006, 44, 15231533.

16. Gohil, B. S. and Pandey, P. C., An algorithm for retrieval of oceanic wind vectors from the simulated SASS normalized radar cross-section measurements. J. Geophys. Res., 1985, 90, 7307-7311.

17. Gohil, B. S., Extraction of ocean surface wind field from simulated ERS-1 scatterometer data. Int. J. Remote Sensing, 1992, 13, 3311-3327.

18. Gohil, B. S., Sarkar, A. and Agarwal, V. K., A new algorithm for wind-vector retrieval from scatterometers. IEEE Geosci. Remote Sensing Lett., 2008, 5, 387-391.

19. Gohil, B. S., Sharma, P., Sikhakolli, R. and Sarkar, A., Directional stability and conservation of scattering (DiSCS) based directionalambiguity removal algorithm for improving wind-fields from scatterometer: a QuikSCAT example. IEEE Geosci. Remote Sensing Lett., 2010, 7, 592-595.

20. Stiles, B. W., Polland, B. D. and Dunbar, R. S., Direction interval retrieval with thresholded nudging: a method for improving the accuracy of QuikSCAT winds. IEEE Trans. Geosci. Remote Sensing, 2002, 40, 79-89.

21. Huddleston, J. N. and Stiles, B. W., Multidimensional histogram rain-flagging technique for sea winds on QuikSCAT. In Proceedings of International Geoscience and Remote Sensing Symposium - 2000, vol. 3, pp. 1232-1234.

22. Stiles, B. W. and Yueh, S. H., Impact of rain on spaceborne Kuband wind scatterometer data. IEEE Trans. Geosci. Remote Sensing, 2002, 40, 1973-1983.

23. Gohil, B. S., Sikhakolli, R., Gangwar, R. K. and Kiran Kumar, A. S., Oceanic rain-flagging using radar backscatter and noise measurements from Oceansat-2 scatterometer. IEEE Trans. Geosci. Remote Sensing, 2016, 54, 2050-2055.

ACKNOWLEDGEMENTS. We thank the Director, Space Applications Centre (SAC; ISRO), Ahmedabad and senior members of the SAC fraternity for their continuous guidance, support and encouragement. We also thank SCATSAT-1 project teams at various ISRO centres for their support in carrying out this study. We also thank anonymous reviewers for their suggestions for improving the quality of the paper.

doi: $10.18520 / \mathrm{cs} / \mathrm{v} 117 / \mathrm{i} 6 / 950-958$ 